\title{
Efecto de Vitamina E y Alendronato sobre la Densidad Mineral en Hueso Maxilar de Ratas
}

\author{
Effect of Vitamin E and Alendronate on Mineral Density in Rat Maxillary Bone
}

\author{
Cecilia Moro; Alejandra Aguzzi \& Carolina Virga
}

MORO, C.; AGUZZI, A. \& VIRGA, C. Efecto de vitamina E y alendronato sobre la densidad mineral en hueso maxilar de ratas. Int. J. Odontostomat. 13(2):137-141, 2019.

RESUMEN: El balance óseo está mediado por una regulación inmunoendócrina, siendo éste un complejo proceso. Entre las acciones llevadas a cabo para mantener la densidad y estructura del esqueleto son variadas las farmacoterapias utilizadas. Diversos estudios han demostrado que tanto Alendronato $(A L)$ y Vitamina $E(E)$ contribuyen a la inhibición de la reabsorción ósea. El objectivo de este trabajo fue estudiar el efecto de la administración combinada de (AL) por vía subcutánea y $(E)$ se administró tres veces por semana también por vía subcutánea con una dosificación de $20 \mathrm{mg} / \mathrm{kg}$ de peso corporal. La fórmula farmacéutica fue de $0,5 \mathrm{mg} / \mathrm{kg}$ de peso corporal para $\mathrm{AL}$, y $20 \mathrm{mg} / \mathrm{kg}$ de vitamina E. El efecto se evaluó en ratas machos Wistar $(\mathrm{n}=108)$, de $90 \pm 20 \mathrm{~g}$, divididas en 4 grupos. Se realizó la exodoncia de los primeros molares inferiores. La droga se inyectó en forma subcutánea en tiempos 0, 7, 15 y 30 días post cirugía. Las imágenes de las mandíbulas fueron adquiridas mediante radiovisiógrafo, en cada tiempo experimental y fueron analizadas con el Software Image ProPlus versión 4,1 de Media Cibernetics. Estudios estadísticos: no paramétrico: prueba de Kruskal-Wallis Resultados: El grupo $\mathrm{C}$ (que registró la media de intensidad más baja), se diferenció significativamente de los grupos $\mathrm{E}$ y A-E $(p<0,001)$, no así del grupo que utilizó únicamente $\mathrm{Al}(\mathrm{p}=0,070 ; p>0,05)$. Los grupos $\mathrm{Al}$, E y el combinado Al-E no se diferenciaron significativamente entre sí ( $p>0,05$ en todos los casos). Los datos evaluados sirven para mostrar una tendencia favorable en relación al efecto beneficioso de la combinación de AL y vitamina $E$.

PALABRAS CLAVE: bisfosfonatos, vitamina E, remodelación ósea.

\section{INTRODUCCIÓN}

El hueso es un tejido dinámico que preserva el tamaño, la forma y la integridad estructural del esqueleto y que regula la homeostasis mineral. La homeostasis ósea se mantiene a través de un equilibrio entre la formación ósea osteoblástica y la resorción ósea osteoclástica (Fernández-TresguerresHernández-Gil et al., 2006).

La regeneración tisular es la respuesta que consigue la restitutio ad integrum del tejido tras un trauma, a diferencia de la reparación, donde el tejido que se forma es un tejido cicatricial, con características diferentes al original. En este sentido el hueso es el único tejido del organismo, a excepción del tejido embrionario, que se restituye totalmente tras una lesión (Van der Weijden et al., 2009).

La vitamina $E(E)$, un antioxidante soluble en grasa, ha sido de particular interés debido a su capa- cidad para suprimir la producción de ciertos mediadores proinflamatorios como PGE2, TNF-a, IL-1 e IL-6 que se han relacionado con el aumento de la pérdida ósea, y proteger contra el daño oxidativo causado por ODFRs. Mantiene el crecimiento óseo normal y el modelado óseo en animales jóvenes y protege el cartílago contra la peroxidación lipídica celular. También encontraron que la $(E)$ suplementaria aumentó la masa ósea disminuyendo las concentraciones de radicales libres que estimulan la resorción ósea y suprimen la formación ósea (Chai et al., 2008; Hamidi et al., 2012).

La vitamina $E$ también redujo la estimulación formación de radicales libres derivados de oxígeno (ODFR) de la resorción ósea osteoclástica. Además, se demostró que la administración de a-tocoferol suprime la producción de ODFR en las primeras etapas de la cicatrización de la fractura en conejos, aumenta la actividad de las enzimas antioxidantes en las ratas 
ovarectomizadas (Ovx) y mejora la curación de la fractura ósea en sus etapas tempranas y finales de las fases en Ovx ratas y en ratas macho. Además, los resultados de un estudio poblacional indicaron el importante papel de la ingesta adecuada de (E) en la dieta para reducir el riesgo de fractura de cadera en los fumadores actuales (Mehat et al., 2010; Feresin et al., 2013).

Los bifosfonatos (BP) son una clase importante de fármacos antirresortivos. BPs se dividen en los que contienen nitrógeno (N-BP) y no-N-BP. La estructura de P-C-P estructura de BP presenta una alta afinidad por el mineral óseo, y por lo tanto BP se acumulan en las superficies óseas. Los osteoclastos internalizan las BP acumuladas durante el proceso de resorción ósea (Coxon et al., 2008; Bobyn et al., 2009).

El análisis molecular de su efecto sobre la resorción ósea reveló que los N-BP tales como alendronato, risedronato y zoledronato inhiben la farnesil difosfato sintasa, una enzima clave de la vía del mevalonato. Posteriormente, previenen la síntesis de isoprenoides requeridos para la prenilación de pequeñas GTPasas tales como Rap, Rac, Rho, Rab y Cdc42. La acumulación de pequeñas GTPasas no preniladas por N-BPs causa disrupción del citoesqueleto de actina, tráfico alterado de componentes intracelulares y alteración de la señalización de integrina en osteoclastos (Bellido \& Plotkin, 2011; Abtahi et al., 2012).

Los hallazgos apoyan la hipótesis de que el alendronato $(A L)$ se une a los sitios de resorción ósea, se libera localmente por acidificación, lo que aumenta su concentración local bajo los osteoclastos e interfiere con la reabsorción ósea y formación de márgenes ondulados. Así mismo la Vitamina funcionaría como un cofactor que mejoraría las condiciones de respuesta del hueso.

\section{MATERIAL Y MÉTODO}

Preparación de las formulaciones: Se preparó una solución de AL para ser aplicada semanalmente por vía subcutánea con una dosificación de 0,5 $\mathrm{mg} / \mathrm{Kg}$ de peso corporal. E se administró semanalmente también por vía subcutánea con una dosificación de $20 \mathrm{mg} / \mathrm{kg}$ de peso corporal. Ambas drogas fueron administradas siempre en el mismo horario (10:00 AM).
Prueba en animales de experimentación: Noventa y seis (96) ratas macho de la línea Wistar de peso 90 $\pm 15 \mathrm{~g}$, se dividieron en 4 grupos de 24 ratas cada uno. Un grupo fue control (C). Recibió semanalmente $1 \mathrm{ml} / 100 \mathrm{~g}$ de peso corporal de solución salina por vía subcutánea. El segundo grupo $(\mathrm{AL})$ recibió semanalmente $0,5 \mathrm{mg}$ de $\mathrm{AL} / \mathrm{Kg}$ de peso corporal por vía subcutánea. El tercer grupo $(\mathrm{E})$ recibió tres veces por semana $20 \mathrm{mg} / \mathrm{kg}$ de peso corporal por vía subcutánea. El cuarto grupo recibió tratamiento combinado con AL y E por vía subcutánea. Un quinto grupo (Control Aceite de Sésamo) fue pautado para actuar como Control de E, pero como se comportó de manera similar al primer grupo $\mathrm{C}$ no fue utilizado.

Los animales se mantuvieron en bioterio en jaulas colectivas con alimento balanceado y agua de bebida ad libitum, a una temperatura de $22-26{ }^{\circ} \mathrm{C}$, con un ciclo luz-oscuridad: $12 \mathrm{~h}-12 \mathrm{~h}$ durante el tiempo que duró el experimento.

El manejo de los animales fue realizado siguiendo las normas establecidas por SECyT según el Comité Institucional para el Cuidado y Uso de Animales de Laboratorio (Res HCD 674/09 Facultad de Ciencias Médicas).

Al inicio del experimento los animales fueron anestesiados con una solución de ketamina/xilazina en una relación $8 \mathrm{mg} / 1,28 \mathrm{mg}$ respectivamente por cada $100 \mathrm{~g}$ de peso corporal. Previa asepsia del campo quirúrgico con iodopovidona se realizó la extracción del primer molar inferior derecho e izquierdo. El alvéolo no fue rellenado con ningún material sino sólo por sus propios coágulos.

Al finalizar el experimento se realizó la eutanasia de los animales mediante inyección intracardiaca de cloruro de potasio, bajo anestesia general. Los sacrificios para la toma de muestras fueron a los $0,7,15$ y 30 días. En todos los tiempos se realizaron tomas radiográficas de la mandíbula.

Estudios radiográficos: Se tomaron imágenes radiográficas de las mandíbulas con radiovisiógrafo (software Sopix SOPRO), en cada tiempo experimental, y se analizaron con el Software Image ProPlus versión 4,1 de Media Cibernetics, diseñado para trabajar con microscopios confocales Olympus. Este software mide densidad óptica (escala de grises) y obtiene valores numéricos que son registros de las siguientes zonas: hueso medular, hueso cortical, zona problema y área circundante a la zona problema. Dicha 
densidad de los diferentes sectores fue cuantificado $\left(\mathrm{mg} \mathrm{Ca}++/ \mathrm{cm}^{2}\right)$ utilizando un patrón de aluminio.

Estudios estadísticos: La comparación de los datos se realizó por análisis de la varianza (ANOVA) a dos criterios de clasificación (tratamientos: $\mathrm{C}, \mathrm{AL}, \mathrm{E}, \mathrm{AL}+\mathrm{E}$, y tiempos de tratamiento: $0,7,15$ y 30 días). Se utilizó como test complementario la prueba de Tukey que realiza comparaciones múltiples entre categorías correspondientes a los factores grupos (tratamientos) y días (tiempos) para determinar la manera en que diferían.

Se consideraron diferencias estadísticamente significativas si $p<0,05$. Para el análisis de datos, construcción de gráficas y análisis estadísticos se utilizó el software (GraphPad Prism 2.0, GraphPad Software, San Diego, CA USA).

\section{RESULTADOS}

La Figura 1 muestra la medición sobre la imagen radiográfica. Se delimitó el área de la exodoncia en donde se midió la radiopacidad promedio. Para ello se tomaron los niveles de intensidad (nivel de gris) de la escala de referencia o testigo de 5 escalones, radiografiada conjuntamente con los maxilares de los especímenes, y se midió la intensidad lumínica promedio de la zona alveolar correspondiente a la exodoncia.

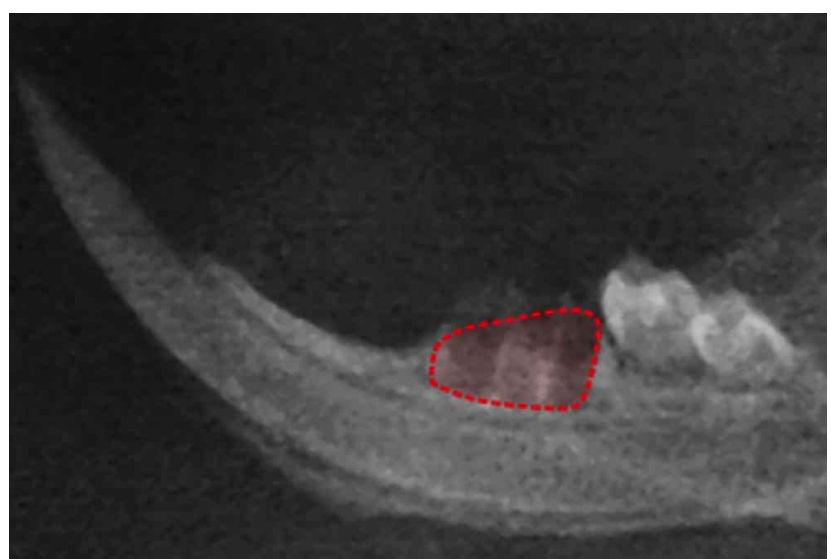

Fig. 1. Esquema de medición sobre imagen radiográfica. Demarcación del área de la exodoncia en donde se midió la radiopacidad promedio.

A los 0 días se registran valores de referencia en todos los grupos. A los 7 días las diferencias entre los grupos son escasas, solo AL-E mostró mayor densidad óptica.
A los 15 días de tratamiento los valores del grupo $C$ y AL fueron similares, en tantos los grupos $E$ y AL-E fueron semejantes entre sí, registrando mayores densidades ópticas que grupos $\mathrm{C}$ y AL: se encontraron diferencias significativas entre grupo $C$ y $A L-E$ $(p<0,001)$ (Fig. 2).

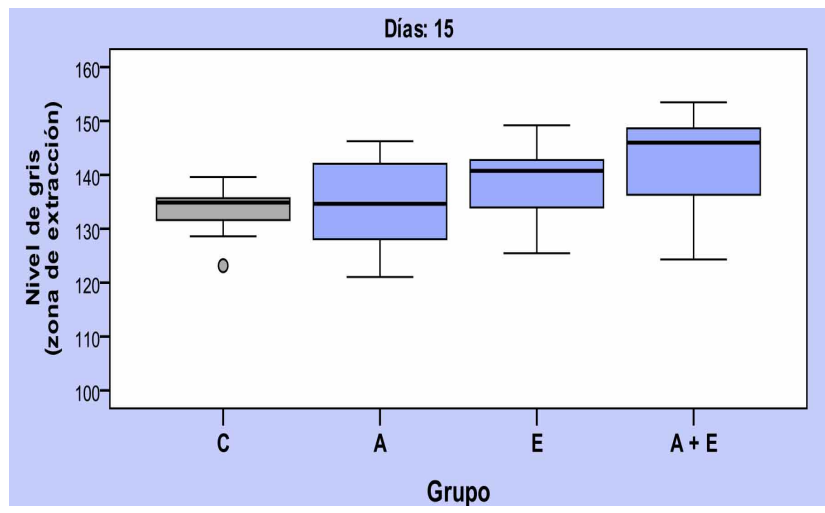

Fig. 2. Distribución esquemática de valores de niveles de grises.

A los 30 días, las distribuciones de intensidad (nivel de gris) de los grupos $\mathrm{E}$ y AL-E mostraron mayor densidad óptica que $C$ y $A L$, con diferencias estadísticamente significativas ( $p<0,001)$ (Fig. 3 ).

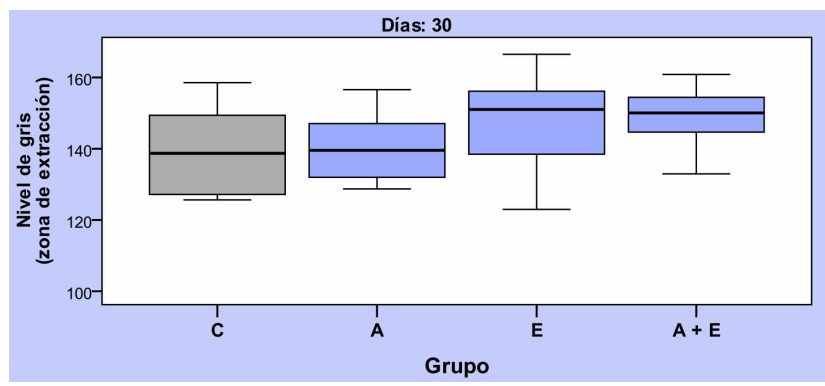

Fig. 3. Distribución esquemática de valores de niveles de grises.

Los niveles de intensidad (nivel de gris) resultaron significativamente diferentes entre todas las etapas con excepción de las últimas dos (15 y 30 días) $(p=0,068 ; p>0,05)$. Entre la etapa inicial y la de 1 semana de tratamiento, las diferencias fueron moderadas pero significativas $(p=0,046 ; p<0,05)$ en tanto que entre 7 y 15 días la diferencia de intensidad entre etapas fue notoria $(p<0,001)$.

\section{DISCUSIÓN}

En el presente estudio, el análisis de la densidad mineral óptica (DMO), por medio de los estudios 
radiográficos, permitió establecer la calidad de hueso formado en el alvéolo post extracción, a través de un aumento de la radiopacidad de esta zona conforme al avance del tiempo. Radiográficamente, se determinó un aumento en la DMO en los grupos experimentales. El valor más significativo fue para Vitamina $E$ más $A L$ a los 30 días.

Por otro lado, Lucisano et al. (2013) analizaron la $\mathrm{DMO}$ en ratas, después de la administración sistémica de $\mathrm{AL}$, utilizando dosis de $1 \mathrm{mg} / \mathrm{kg}$ por semana. En su trabajo demostraron una DMO significativamente mayor que el grupo control recién después de 8 semanas de tratamiento (60 días). La comparación de los grupos con y sin tratamiento con AL reveló un aumento de un $14,9 \%$ y $26 \%$ en la DMO.

Vitamina E, a-tocoferol, es un derivado poliisoprenoide con actividades antioxidantes que se comporta como un verdadero secuestrador de radicales libres. Sin embargo, el verdadero efecto de $\mathrm{E}$ en el hueso es incierto. Una revisión de los estudios sobre el efecto de tocoferoles en la reparación de fracturas sugiere que Vitamina $\mathrm{E}$ puede afectar la remodelación ósea y la curación debido a sus propiedades antioxidantes, pero citó la necesidad de más datos para ser recolectados (Borhanuddin et al., 2012). En un estudio utilizando ratones genéticamente incapaces de importar Vitamina $\mathrm{E}$, los ratones mostraron disminución de masa ósea debido al aumento de la fusión de osteoclastos. Tras la alimentación de tocoferoles a estos ratones, se restauró un fenotipo de masa ósea normal y la fusión de osteoclastos se mantuvo elevada (Fujita et al., 2012). A pesar de estos hallazgos, en un estudio con pérdida ósea inducida por ovariectomía, Vitamina $\mathrm{E}$ a $60 \mathrm{mg} / \mathrm{kg}$ por día y tocotrienol puro a la misma dosis fueron completamente protectores (Muhammad et al., 2012).

De la misma manera, en nuestros estudios radiográficos se pudo observar que a partir de los 15 días se evidencia un aumento de la densidad mineral ósea a favor del grupo de Vitamina $E$ y AL-E, siendo mayor para éste último grupo. A los 30 días se observan diferencias más definidas con la misma tendencia anterior.

Se ha demostrado que los niveles suplementarios de vitamina $\mathrm{E}$ mejoran la calidad del hueso, así como aumentan la proteína de la matriz ósea sin alterar la densidad ósea en ratones machos, ambos modelos bien aceptados. Además, se observó que las dosis suplementarias de vitamina $E$ tienen efectos pro- tectores sobre diversos parámetros óseos en el modelo de rata macho (Shuid et al., 2010; Iwaniec et al., 2013).

Así mismo, en nuestro trabajo, una dosis de Vitamina E $20 \mathrm{mg} / \mathrm{kg}$ de peso corporal administrada semanalmente demostró ser eficaz para mejorar la densidad mineral ósea luego de la extracción del primer molar en ratas macho.

\section{CONCLUSIONES}

Radiográficamente se observó un incremento en la densidad mineral óptica de los grupos vitamina E y vitamina $E$ más Alendronato con respecto al control conforme avanza el tiempo, siendo más evidentes los 30 días.

Por lo anteriormente expuesto se puede inferir que, teniendo en cuenta la variable estudios radiográficos, tanto la $\mathrm{E}$ como la combinación de $\mathrm{AL}$ $E$ ejercerían un efecto beneficioso produciendo un aumento en la remodelación ósea de alvéolos post exodoncia. Este efecto es netamente benéfico en la práctica odontológica, donde el hueso constituye el principal sostén de los elementos dentarios y de estructuras de titanio cuando aquellos se pierden por distintas patologías y también formando parte esencial del sistema estomatognático, para que este pueda cumplir con eficiencia todas sus funciones.

MORO, C.; AGUZZI, A. \& VIRGA, C. Effect of vitamin E and alendronate on mineral density in rat maxillary bone. Int. J. Odontostomat., 13(2):137-141, 2019.

ABSTRACT: The bone balance is mediated by an immunoendocrine regulation, this being a complex process. A number of pharmacotherapies are used among the actions taken to maintain the density and structure of the skeleton. Several studies have shown that both Alendronate $(A L)$ and Vitamin $E(E)$ contribute to the inhibition of bone resorption. Objective: To study the effect of combined administration of (LA) subcutaneously and (E) was administered three times per week also subcutaneously with a dosage of $20 \mathrm{mg} / \mathrm{kg}$ body weight. The pharmaceutical formulation was $0.5 \mathrm{mg} /$ $\mathrm{kg}$ body weight for $\mathrm{AL}$ and $20 \mathrm{mg} / \mathrm{kg}$ vitamin $\mathrm{E}$. The effect was evaluated in male Wistar rats $(n=108), 90 \pm 20 \mathrm{~g}$, divided into 4 groups. Extraction of the first lower molars was performed. The drug was injected subcutaneously at time 0 , 7,15 and 30 days post-surgery. The images of the jaws were acquired by radiovisiography, at each experimental time and 
were analyzed with Image ProPlus Software version 4.1 of Media Cibernetics. Statistical studies: non-parametric: Kruskal-Wallis test Group C (which recorded the lowest mean intensity) was significantly different from the $E$ and $A E$ groups $(p<0.001)$, but not from the group that used only $\mathrm{Al}(\mathrm{P}=$ $0.070, p>0.05)$. The Al, E and combined Al-E groups did not differ significantly from each other ( $p>0.05$ in all cases). The data evaluated serve to show a favorable trend in relation to the beneficial effect of the combination of $A L$ and vitamin $E$. remodeling.

KEY WORDS: bisphosphonates. vitamin E. bone

\section{REFERENCIAS BIBLIOGRÁFICAS}

Abtahi, J.; Tengvall, P. \& Aspenberg, C. A bisphosphonate-coating improves the fixation of metal implants in human bone. A randomized trial of dental implants. Bone, 50(5):1148-51, 2012.

Bellido, T. \& Plotkin, L. I. Novel actions of bisphosphonates in bone: preservation of osteoblast and osteocyte viability. Bone, 49(1):50-5, 2011.

Bobyn, J. D.; McKenzie, K.; Karabasz, D.; Krygier, J. J. \& Tanzer, M. Locally delivered bisphosphonate for enhancement of bone formation and implant fixation. J. Bone Joint Surg. Am., 91 Suppl. 6:23-31, 2009.

Borhanuddin, B.; Fozi, N. F. M. \& Mohamed, I. N. Vitamin E and the healing of bone fracture: the current state of evidence. Evid. Based Complement. Altern. Med., 2012:684510, 2012.

Chai, S. C.; Wei, C. I.; Brummel-Smith, K. \& Arjmandi, B. H. The role of vitamin $\mathrm{E}$ in reversing bone loss. Aging Clin. Exp. Res., 20(6):521-7, 2008

Coxon, F. P.; Thompson, K.; Roelofs, A. J.; Ebetino, F. H. \& Rogers, M. J. Visualizing mineral binding and uptake of bisphosphonate by osteoclasts and non-resorbing cells. Bone, 42(5):848-60, 2008.

Feresin, R. G.; Johnson, S. A.; Elam, M. L.; Kim, J. S.; Khalil, D. A.; Lucas, E. A.; Smith, B. J.; Payton, M. E.; Akhter, M. P.; et al. Effects of vitamin $E$ on bone biomechanical and histomorphometric parameters in ovariectomized rats. J. Osteoporos., 2013:825985, 2013.

Fernández-Tresguerres-Hernández-Gil, I., Alobera-Gracia, M. A.; del-Canto-Pingarrón, M. \& Blanco-Jerez, L. Physiological bases of bone regeneration I. Histology and physiology of bone tissue. Med. Oral Patol. Oral Cir. Bucal, 11(1):E47-51, 2006.

Fujita, K.; Iwasaki, M.; Ochi, H.; Fukuda, T.; Ma, C.; Miyamoto, T.; Takitani, K.; Negishi-Koga, T.; Sunamura, S.; Kodama, T.; et al. Vitamin $\mathrm{E}$ decreases bone mass by stimulating osteoclast fusion. Nat. Med., 18(4):589-94, 2012.

Hamidi, M. S.; Corey, P. N. \& Cheung, A. M. Effects of vitamin E on bone turnover markers among US postmenopausal women. J. Bone Miner. Res., 27(6):1368-80, 2012.

Iwaniec, U. T.; Turner, R. T.; Smith, B. J.; Stoecker, B. J.; Rust, A.; Zhang, B.; Vasu, V. T.; Gohil, K.; Cross, C. E. \& Traber, M. G. Evaluation of long-term vitamin $E$ insufficiency or excess on bone mass, density, and microarchitecture in rodents. Free Radic. Biol. Med., 65:1209-14, 2013.

Lucisano, M. P.; Nelson-Filho, P.; Morse, L.; Battaglino, R.; Watanabe, P. C.; Silva, R. A. \& Silva, L. A. Radiodensitometric and DXA analyses for the measurement of bone mineral density after systemic alendronate therapy. Braz. Oral Res., 27(3):252$7,2013$.
Mehat, M. Z.; Shuid, A. N.; Mohamed, N.; Muhammad, N. \& Soelaiman, I. N. Beneficial effects of vitamin E isomer supplementation on static and dynamic bone histomorphometry parameters in normal male rats. J. Bone Miner. Metab., 28(5):5039, 2010.

Muhammad, N.; Luke, D. A.; Shuid, A. N.; Mohamed, N. \& Soelaiman, I. N. Two different isomers of vitamin e prevent bone loss in postmenopausal osteoporosis rat model. Evid. Based Complement. Alternat. Med., 2012:161527, 2012.

Shuid, A. N.; Mehat, Z.; Mohamed, N.; Muhammad, N. \& Soelaiman, I. N. Vitamin E exhibits bone anabolic actions in normal male rats. J. Bone Miner. Metab., 28(2):149-56, 2010.

Van der Weijden, F.; Dell'Acqua, F. \& Slot, D. sE. Alveolar bone dimensional changes of post-extraction sockets in humans: a systematic review. J. Clin. Periodontol., 36(12):1048-58, 2009.

Dirección para correspondencia:

Dra. Alejandra Aguzzi

Profesora Titular

Cátedra de Farmacología y Terapéutica A

Facultad de Odontología

Universidad Nacional de Córdoba

Córdoba

ARGENTINA

Email: aleceagu@yahoo.com.ar

Recibido :30-07-2018

Aceptado:28-02-2019 